\title{
A Micromechanics model for Bread Dough
}

\author{
M.A. P Mohammed, E. Tarleton, M.N. Charalambides, J.G. Williams
}

Imperial College London, Mechanical Engineering Department, London SW7 2AZ, UK

\begin{abstract}
The mechanical behaviour of dough and gluten was studied in an effort to investigate whether bread dough can be treated as a two phase (starch and gluten) composite material. The dough and gluten show rate dependent behaviour under tension, compression and shear tests, and non-linear unloading-reloading curves under cyclic compression tests. There is evidence from cryo-Scanning Electron Microscopy (SEM) that damage in the form of debonding between starch and gluten occurs when the sample is stretched. A composite finite element model was developed using starch as filler and gluten as matrix. The interaction between the starch and gluten was modelled as cohesive contact. The finite element analysis predictions agree with trends seen in experimental test data on dough and gluten, further evidence that debonding of starch and gluten is a possible damage mechanism in dough.
\end{abstract}

Keywords: Dough, Gluten, Composite model, Visco-hyperelastic

PACS: 83.80.Ya, 83.60.Df, 83.85.Rx, 68.37.Hk

\section{INTRODUCTION}

Bread dough is a simple mixture of wheat flour, salt and water; however its mechanical behaviour is surprisingly complex. Bread dough behaves as a viscoelastic material. The two major components of dough which are believed to influence its mechanical properties are starch and gluten. These two phases interact by forming starch-starch, starch-gluten or gluten-gluten interactions [1]. The interactions store potential energy upon deformation and thus contribute to the elastic behaviour of the dough.

Dough can be modelled as a composite material, where starch is modelled as a harder filler and gluten as a softer matrix. However studies on the stress-strain behaviour of dough and gluten showed that gluten is stronger than dough at large strain [2]. This is in contrast with the composite material theory, where in the case of a stiffer filler, the composite material is expected to be stiffer than its matrix. It is believed that the reason for this is due to damage and debonding of the starch-gluten interface. In a weakly bound filler-matrix composite material, the filler-matrix interaction will start to damage and eventually debond at a certain value of energy release rate (see Figure 3) [3]. Therefore this work investigates the possibility that damage at the interface between the dough and gluten could explain the stress-strain characteristics of dough.

\section{MECHANICAL TESTS AND MICROSTRUCTURE OF DOUGH AND GLUTEN}

Wheat flour dough was prepared using the procedures by Charalambides et al. [4]. The flour used is strong white bread flour purchased from Wessex Mill in Oxford, United Kingdom. A mixture of $198.5 \mathrm{~g}$ of wheat flour, $120 \mathrm{~g}$ of distilled water and $1.5 \mathrm{~g}$ of sodium chloride is used to make the dough $(62 \%, 37.5 \%$ and $0.5 \%$ of wheat flour, water and salt respectively). The mixture was mixed at the speed of $118 \mathrm{rpm}$ for two minutes. The dough was then washed under running tap water and was gently rubbed manually to remove the starch from the dough. The starch was assumed to be absent when no cloudiness appeared after the gluten was squeezed into a container of clear water. Mechanical tests under tension, compression, and cyclic compression modes were performed on the dough and gluten samples using the Instron 5543 with a $100 \mathrm{~N}$ load cell. Shear tests were performed using the rheometer model TA2000ex. Cryo-SEM tests were performed on dough samples mixed from the same batch. The samples were stretched and compressed manually, and were compared with the unstretched sample. The samples were slushed in liquid nitrogen under vacuum conditions before being transferred to the cryo chamber. The samples were then freeze-fractured, sublimated at $-90^{\circ} \mathrm{C}$ for 2 minutes and gold-sputtered before being imaged in the SEM chamber. 
The results of the mechanical tests on the dough and gluten are shown in Figure 1.

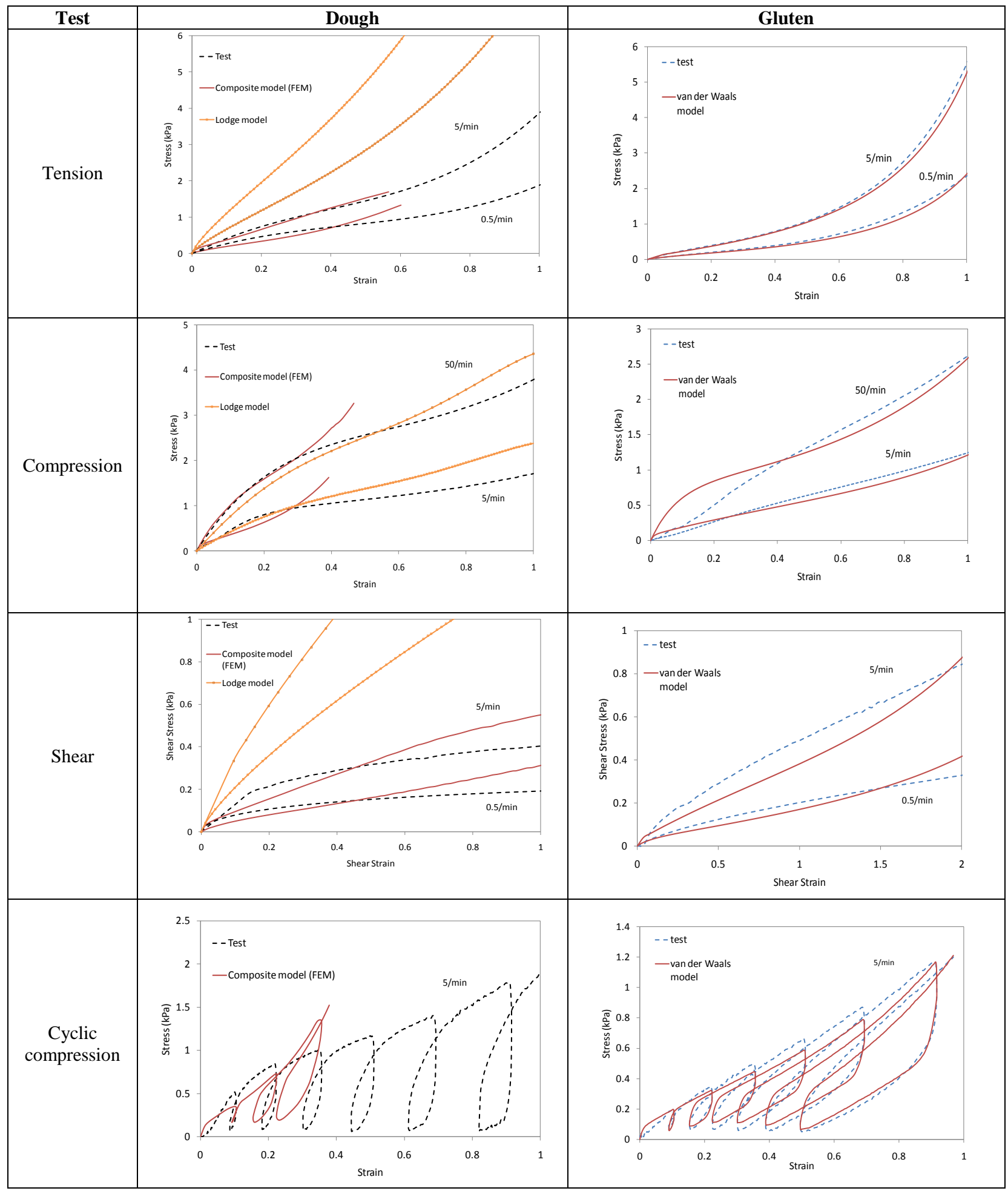

Figure 1. Dough and gluten tests results and modelling. The parameters used for gluten are: van der Waals: $\mu=3.287 \mathrm{kPa}$, $\lambda_{m}=4.64$ and $a=0.25$; Prony series: $0.867,0,0.092,0.004,0.028$ for $g_{1}$ to $g_{5}$ and 0.007 for $g_{\infty}$. The time constants $\tau_{1}$ to $\tau_{5}$ are set at $0.1,1,10,100$ and 1000 seconds. 
They show rate dependent behaviour under tension, compression and shear tests, and non-linear unloadingreloading curves under cyclic compression tests. The results of the cryo-SEM tests are shown in Figure 2. The starch appears as discrete ellipsoidal granules embedded in the gluten matrix. No starch granules are observed on the surface of the gluten sample, as shown in Figure 2b. Evidence of debonding at the starch-gluten interface is observed for the stretched sample (Figure 2d), such debonding was not present in the original sample (Figure 2a). This suggests that the dough may be undergoing damage [5] due to the starch-gluten interaction weakening at large deformations. It is worth noting that no debonding is apparent when the dough was subjected to a compressive load (Figure 2c).
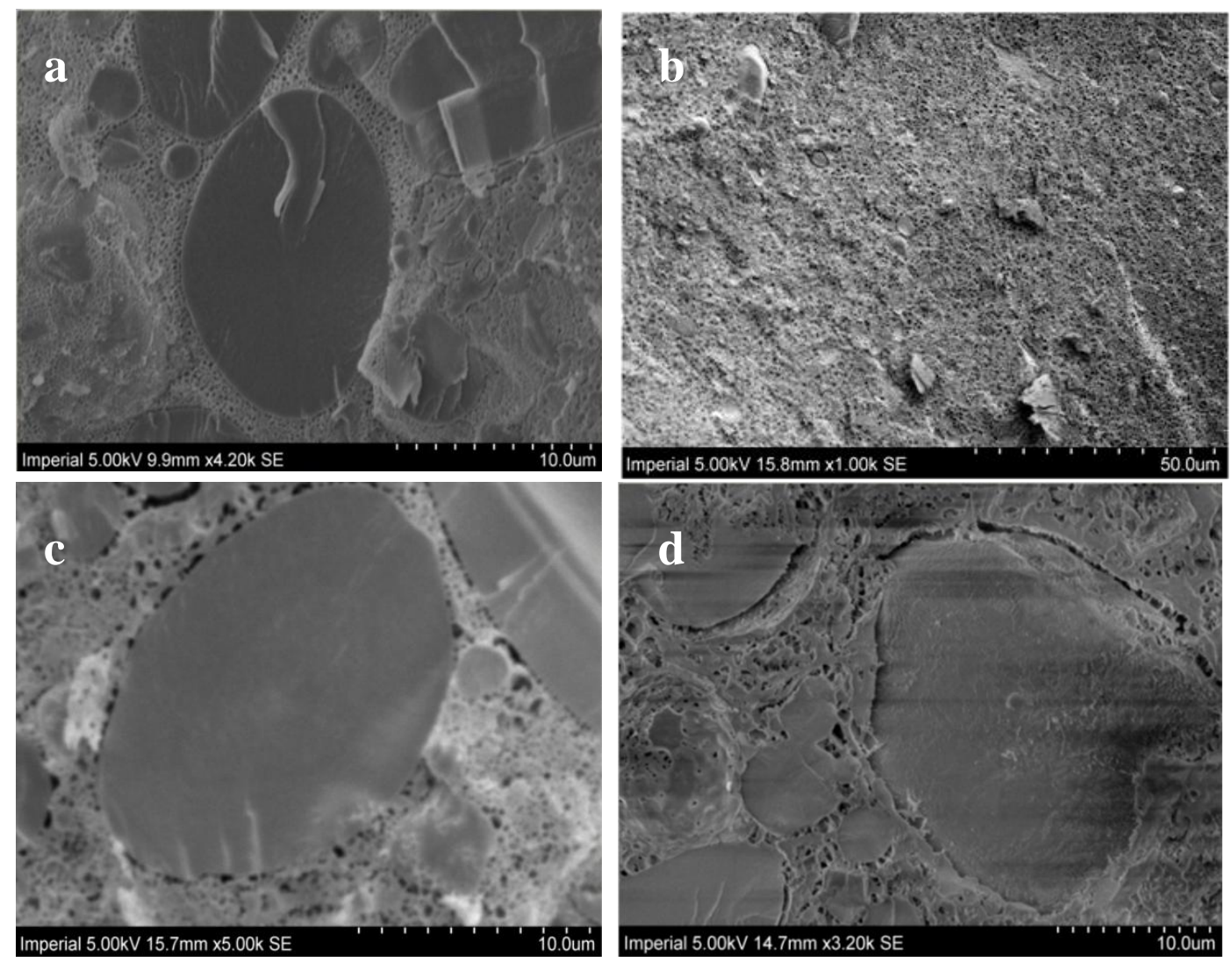

Figure 2. Microstructure of: a) undeformed dough; b) undeformed gluten washed under running tap water; c) compressed dough; and d) stretched dough.

\section{CONSTITUTIVE MODELLING OF DOUGH AND GLUTEN}

The stress-strain data for dough shown in Figure 1 were first used to calibrate the Lodge model [5], the derived material parameters were: $G(1)=2.822 \mathrm{kPa}-s^{n}$ and power law constant, $n=0.22$. Figure 1 shows the fit of the Lodge model to the dough test data. The model leads to much higher stress values than the tension and shear test data, therefore suggesting the use of a damage function [5]. However, the model agrees reasonably well with the compression test data, indicating that possibly no damage occurs under compression.

To investigate the interaction between the starch and gluten, a simple composite finite element model was developed using Abaqus. Simulations of a 2D circular granule represented using generalised plane strain elements were performed. The model consists of a single filler representing starch embedded in a matrix representing the gluten. Average starch volume fraction values of $27 \%, 24.77 \%$ and $29.89 \%$ were calculated from a few cryo-SEM images of dough taken at magnifications of 300x, 500x and 1000x respectively and this was used to determine the relative size of the filler. Starch was modelled as a linear elastic material, whereas gluten was modelled as viscohyperelastic material. A separable time and strain dependent material behaviour was assumed [6], where the relaxation stress under a step strain loading history can be factored into a function of time and a function of strain: 
$\sigma(\varepsilon, t)=f(\varepsilon) g(t)$. The time function is represented as the Prony series: $g(t)=g_{\infty}+\sum_{i=1}^{N} g_{i} \exp \left(-\frac{t}{\tau_{i}}\right)$, with $g_{\infty}$ and $g_{i}$ being spring and damper constants, $t$ is time, and $\tau_{i}$ are time constants. The strain function is represented by the van der Waals hyperelastic model. The visco-hyperelastic model was calibrated using all the gluten test data in Figure 1. The model agrees well with the gluten test results, suggesting a rubberlike behaviour for gluten.

The interaction between starch and gluten was modelled using cohesive contact interaction. The interaction is defined by the traction versus separation behaviour, which can be separated into two regions. In the first region, the traction-separation model assumes a linear elastic behaviour. This is followed by the initiation of damage defined by a normal or shear stress in the second region. Progressive damage in the interface occurs until complete failure. The damage evolution law describes the rate at which the cohesive stiffness is degraded once the damage initiation criterion is reached. The cohesive contact parameters are shown in Figure 3c. These were chosen such that the model results were close to the data from the tension tests on dough performed at $5 / \mathrm{min}$ shown in Figure 1 and $3 \mathrm{a}$. The elastic modulus for starch was assumed to be $80 \mathrm{kPa}$.

Figure 3 shows the composite model results under tensile loading at 5/min for the cases of (i) perfect bonding between filler and matrix (i.e. no damage), (ii) cohesive contact interaction at the interface (i.e. with damage). The tensile results for the gluten are also shown in the same figure for comparison purposes. When the interaction of starch and gluten is strong, as indicated for the dough with no damage, the stress-strain curve is always higher than the gluten. The same set of parameters was used in simulation of compression and shear loading as well as for tension at $0.5 / \mathrm{min}$. The simulation results are compared to the experimental data in Figure 1 . Even though the numerical model is rather simple and the starch properties have not been verified experimentally so far, the agreement seems reasonable.

a)

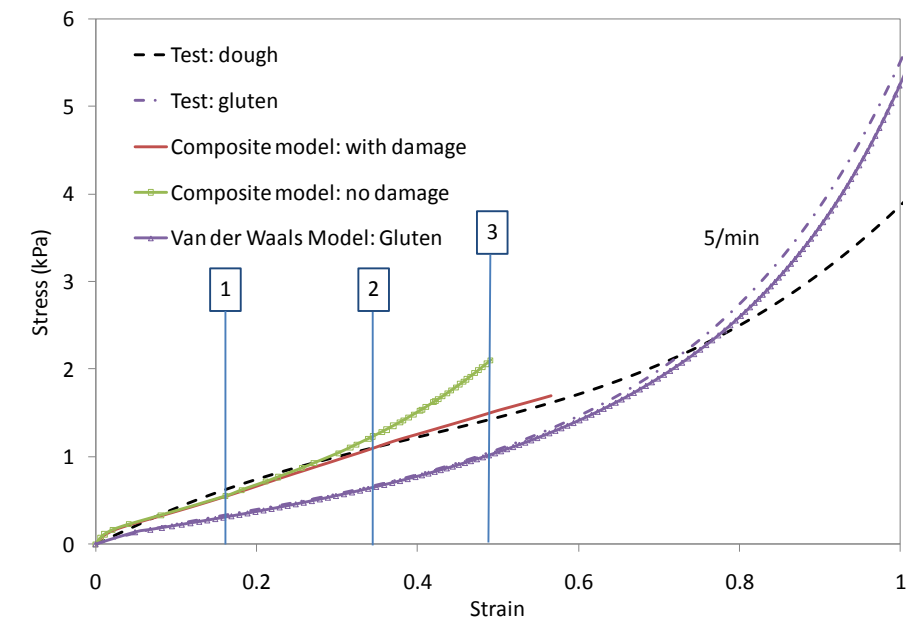

c)

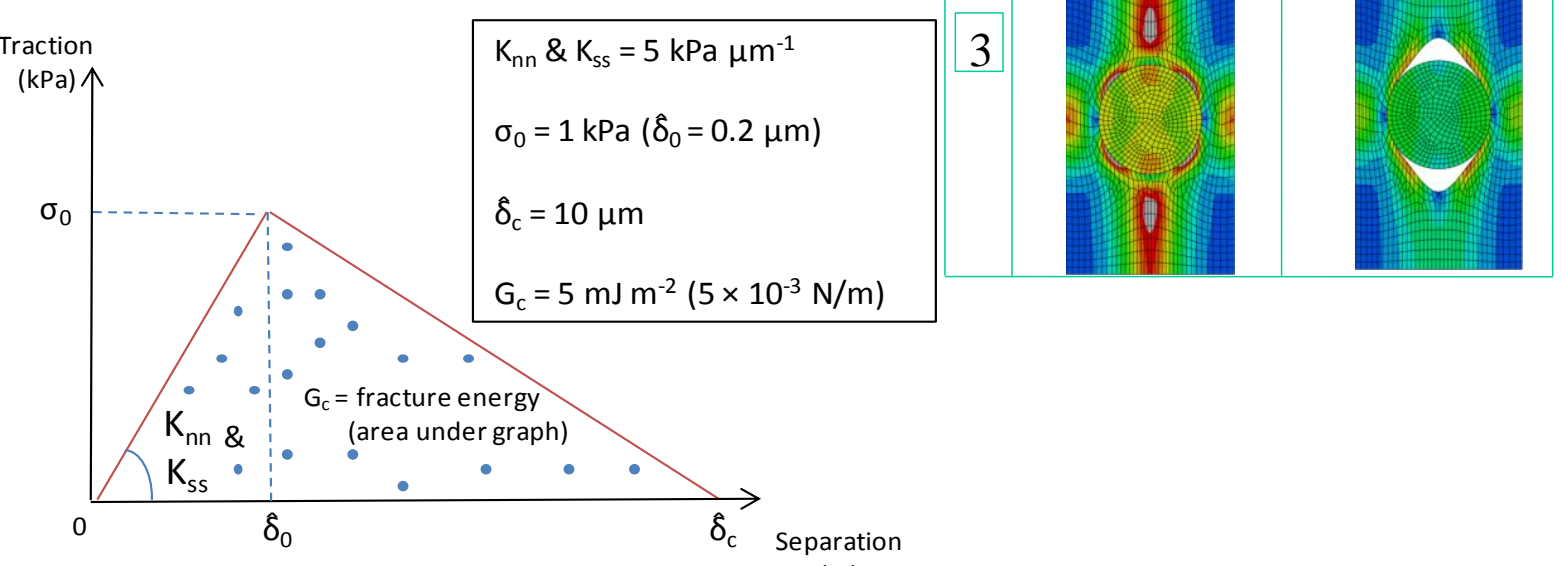

(m)

Figure 3. a) and b) simulation of composite model performed at 5/min in tension; and c) parameters for cohesive contact. 


\section{CONCLUSIONS}

The mechanical tests on gluten showed that gluten behaves as rate-dependent, rubberlike, material. A composite model is developed in Abaqus using starch as a filler and gluten as the matrix. Starch is modelled as a linear elastic material, whereas gluten is modelled as a visco-hyperelastic material. The interaction between the starch and gluten is modelled using cohesive contact. The simple composite model agrees with the results from mechanical tests on dough, indicating possible debonding of starch and gluten in dough under tension and shear. This argument is supported further by cryo-SEM images of stretched dough as well as the apparent need of damage functions when the Lodge constitutive model is used to represent the mechanical test data of dough.

\section{ACKNOWLEDGMENTS}

Financial support for this work is provided by the Malaysian Ministry of Higher Education and Universiti Putra Malaysia. Abaqus was provided under academic licence by HKS Inc., Providence, Rhode Island, USA.

\section{REFERENCES}

1. J.I. Amemiya and J.A. Menjivar, J. of Food Engineering 16, 91-108 (1992).

2. S. Uthayakumaran, M. Newberry, N. Phan-Thien and R.I. Tanner, Rheologica Acta 41, 162-172 (2002).

3. A. Meddad and B. Fisa, J. of Applied Polymer Science 65, 2013-2024 (1997).

4. M.N. Charalambides, L. Wanigasooriya, J.G. Williams, S.M. Goh and S. Chakrabarti, Rheologica Acta 46, $239-248$ (2006).

5. R.I. Tanner, F. Qi and S.C. Dai, J. Non-Newtonian Fluid Mech. 148, 33-40 (2008).

6. S.M. Goh, M.N. Charalambides and J.G. Williams, Mech. Of Time-Dependent Behaviour 8, 255-248 (2004). 